\title{
Short-term variation in ocular anterior chamber angle using Scheimpflug photography
}

\author{
A Rubin*
}

Department of Optometry, University of Johannesburg, PO Box 524, Auckland Park, 2006 South Africa

<arubin@uj.ac.za>

Received 23 November 2009; revised version accepted 5 March 2010

\begin{abstract}
Purpose: To investigate short-term variation of ocular Anterior Chamber Angle (ACA) within and across the different meridians of young, healthy eyes.
\end{abstract}

Method: Scheimpflug photography (via an $\mathrm{Ocu}$ lus Pentacam) was used to repeatedly measure the anterior segment of the right eyes of three young females, all aged 22 years. For each individual, ten consecutive maps were determined over a short time period and each map contained information for the chamber angles of different meridians across the eye involved, roughly at $7^{0}$ intervals. Each meridian in an eye was thus measured 10 times in total permitting analysis of short-term variation both within and across the meridians concerned. Parametric and non-parametric statistical methods used to understand short-term variation of ACA included meridional plots of medians and lower and upper quartiles $\left(Q_{1}\right.$ and $\left.Q_{3}\right)$ and various polar plots (of raw observations, of means and 95\% confidence regions, and of standard deviations).

Results: Over, at least, short periods of time, Scheimpflug photography provided fairly repeatable results for ACA both within and across the meridians of the eyes involved. However, occasionally outliers increased variability for some meridians. While short-term variation within meridians was relatively small assuming exclusion of a limited number of outliers, variation across the meridians of specific eyes was greater (probably relating to the usual anatomical structure of eyes with ACA in the inferior quadrants being mostly larger than that for the superior quadrants). Friedman ANOVA confirmed for each of the three eyes concerned that median ACA was significantly different across the various meridians.

Conclusion: Scheimpflug photography allows for comprehensive, non-invasive and rapid measurement of ACA through $360^{\circ}$. Repeatability of measurements appears satisfactory over the short-term, despite potential limitations relating to this photographic method for measuring ACA. The holistic and quantitative approach to ACA used here may be useful also in diabetic, neoplastic or other disease of the anterior segment of the eye. This methodology could provide better understanding of the potential risk for acute or chronic angle-closure glaucoma through more complete evaluation of ACA. The methods also could be usefully applied to measurement of ACA using techniques such as anterior segment optical coherence tomography or ultrasound biomicroscopy. (S Afr Optom 2010 69(1) 35-47)

Key words: anterior chamber angle, variation of ocular anterior chamber, anterior chamber of the eye, ophthalmic biometry, Scheimpflug photography, Pentacam 


\section{Introduction}

Assessment and quantitative measurement of the ocular anterior chamber angle (ACA) is important particularly where early diagnosis of conditions such as acute or chronic primary angle closure ${ }^{1}$ may be relevant. Worldwide, primary angle-closure glaucoma is a leading cause of preventable blindness ${ }^{2}$. But measurement of ACA is also interesting in terms of underlying processes or conditions that could influence the angle and possibly the dynamics of aqueous outflow. For example, the use of diagnostic drugs such as mydriatics and cycloplegics can have temporary but important effects on the anterior chamber angle $^{2}$. Amongst conditions relevant to the anterior segment of the eye are various pigmentary glaucomas and the additional presence of narrowing or even peripheral regions of closure, such as with peripheral anterior synechiae, could further aggravate the situation. Processes such as accommodation may produce changes in the axial anterior chamber depth or angle itself $^{3}$. There are also many reasons for assessing the anterior chamber even within healthy eyes and, for example, diurnal or other temporally-related changes in ACA are important issues. Such considerations become perhaps even more critical in the presence of diseases such as glaucoma or in relation to surgical procedures such as phaco-emulsification and implantation of intraocular lenses.

The ocular anterior chamber angle can be evaluated via several methods including gonioscopy, ultrasound biomicroscopy (UBM), anterior segment optical coherence tomography (AS-OCT) and Scheimpflug photography (SP) ${ }^{2}$. This paper concentrates on the last of these procedures and uses an Oculus Pentacam incorporating Scheimpflug photography. Although gonioscopy is often regarded as the golden standard for assessment of ACA, the procedure has substantial inter-observer variability ${ }^{2}$, is invasive in nature and requires mainly subjective estimation of angle width in comparison with the other methods as mentioned above. As such gonioscopy can have serious limitations for use in various research-related activities but some investigators have attempted to improve or modify standard gonioscopy to allow for more objective measurement of ACA, for example, through the use of graticules within biomicroscopes that facilitate measurement of angle width (see Congdon et $\mathrm{al}^{4}$, for example). Other concerns include decentered position or excessive pressure on the eye with some lenses used in gonioscopy that could result in either under- or over-estimation of angle structures. Even the level of ambient luminance is important and significant changes to angle size can occur when luminance is changed from bright to dim levels ${ }^{2}$. Of course, the ambient luminance levels are relevant not only to gionioscopy but also to other procedures typically used in ACA assessment. Clinically gonioscopy probably should be performed under dim or even dark conditions to provoke maximal pupil dilation and allow for evaluation of the angles under these somewhat less favourable conditions where dark-induced changes in ocular pupil diameter and the peripheral iris might demonstrate regions of potential narrowing of the ACA that could be relevant.

In comparison with gonioscopy, ultrasound biomicroscopy generally allows for more objective determination of ACA and linear quantities such as angle opening distance (AOD) can be estimated ${ }^{2,5}$. For AOD, imagine a small triangle in one meridian or part of the anterior chamber angle. The triangle naturally consists of three short line segments, and two of them extend onto the anterior, but peripheral, iris and also the posterior and peripheral corneal surface. The third line of the triangle (the AOD) is then the linear distance from the first line (parallel to the posterior corneal surface) to the second line on the anterior iris surface. That is, at the periphery of the anterior chamber, the AOD for any meridian is the distance between the back of the cornea and the front of the iris. The AOD is usually given in micrometres and interested readers should see Pavlin, Harasiewicz and Foster ${ }^{5}$. One limitation of this method is where exactly to place the line (the AOD) between the posterior cornea and anterior iris and Pavlin and colleagues recommended using the trabeculae meshwork (about $250 \mu \mathrm{m}$ anterior to the scleral spur) as a suitable reference point. At this position the mean and standard deviation for the AOD were $347 \pm 158 \mu \mathrm{m}$ (by Pavlin, Harasiewicz and Foster ${ }^{5}$ ). Modern instruments for UBM may determine AOD automatically or allow for the use of cursors or callipers to subjectively measure such quantities but measurements may be influenced by factors such as variation in image acquisition, alignment inconsistencies and ambient luminance ${ }^{2,5}$. UBM probably remains somewhat underutilized due to significant costs involved with the technology and 
other issues such as immersion of the eye that may cause inconvenience when using the procedure.

Recently anterior segment optical coherence tomography (AS-OCT) has become important for imaging the $\mathrm{ACA}^{2}, 6$. With AS-OCT reflected light and interferometry are used to measure tissue depth. Possible disadvantages of AS-OCT are that the technology is expensive and, for instance, in comparison with procedures such as gonioscopy, AS-OCT has a higher false positive rate (eyes that have open angles are sometimes misdiagnosed as having narrow angles) but to its advantage AS-OCT is non-invasive and can be performed rapidly ${ }^{2}$. Xu et al $l^{6}$ used OCT in a large number $(N=2985)$ of Chinese subjects and found mean ACA of $38.3 \pm 16.30^{\circ}$. They measured ACA in the nasal and temporal quadrants only and their subjects ranged in age from 40-101 years with a mean age of 56.2 years. These researchers ${ }^{6}$ also found in their Chinese subjects that narrow angles were associated with age, females, greater hyperopia, short body stature, nuclear cataract, large optic discs and angle closure glaucoma. (Similar associations probably also apply to other races besides the Chinese.)

Wirbelauer et $a l^{7}$ also used AS-OCT for goniometry, particularly measurement of ACA and AOD, and in 138 eyes (of individuals in the range, 23-90 years) found a mean ACA of $28 \pm 16^{\circ}$. The sensitivity and specificity for ACA to predict angles that could be subject to closure was good at 86 and $95 \%$ respectively $^{7}$ suggesting the value of AS-OCT for early diagnosis and implementation of preventative measures in eyes possibly predisposed to angle closure.

Other procedures besides the above-mentioned ones for measuring ACA include the (limbal) van Herick method and scanning peripheral anterior chamber depth analysis (SPAC) ${ }^{2,8}$. The aforementioned procedure, while simple and non-invasive, is also quite subjective and thus somewhat limited for research activities while the second procedure uses slit-lamp based scanning photography, a charge-coupled device (CCD) and a peripheral computer with applicable software. SPAC will require further research prior to wider and more extensive use in angle assessment ${ }^{2}, 8$.

The present study is based on determining ACA using Scheimpflug photography of the anterior segment. (The instrument used here is the Oculus Pentacam but instruments are available from other manufacturers such as Topcon or Nidek.) A possible limitation of the use of SP is that the ACA needs to be photographed through the cornea and the whole angle may not be fully visible ${ }^{2}$ and so some have argued that the procedure has a relatively low correlation with other methods such as gonioscopy or ultrasound biomicroscopy2,9. But there do not appear to be many papers that have used SP or the Pentacam to measure ACA and most papers using SP mainly concentrate on axial anterior chamber depth rather than ACA. For example, Lackner, Schmidinger and Skorpik address issues such as validity and repeatability of measurements of anterior chamber depth with the Pentacam in comparison to the Orbscan ${ }^{10}$. Another study by Buehl et al compares three different methods (Pentacam, Orbscan and AC-Master) for measuring corneal thickness and anterior chamber depth ${ }^{11}$. We will not discuss these papers any further but they suggest that the different methods do provide similar results but whether this would also be true for ACA rather than corneal thickness or anterior chamber depth remains uncertain.

According to Rabsilber, Khoramnia and Auffarth $^{12}$ in 79 healthy volunteers (mean age $46.6 \pm 16.8$ years) the mean anterior chamber angle was 34.81 $\pm 5.050^{\circ}$. The minimum ACA in their sample was $29.99 \pm 5.530^{\circ}$. They studied one eye per volunteer and three measurements per eye were obtained using the Pentacam. They also found that increasing age was associated with reduced $\mathrm{ACA}^{12}$. Shankar et al ${ }^{13}$ also investigated the repeatability of anterior segment biometry using the Pentacam. Two different observers measured both eyes of 35 normal volunteers twice on the same day and the researchers concluded that repeatability was good for several measured parameters ${ }^{13}$, but for anterior chamber angle the relative repeatability (RR) was only $14.41 \%$. The larger the percentage the worse the repeatability and ACA was less repeatable than other parameters as measured using the Pentacam ${ }^{13}$. Recently other studies ${ }^{14-17}$ used the Pentacam to measure ACA and/or anterior chamber depth (ACD) in the context of various clinical disorders such as keratoconus ${ }^{14}$, pseudophakia ${ }^{15}$ and following ocular lens implantation ${ }^{16,17}$. ACA was found to vary according to severity of keratoconus ${ }^{14}$. Using AS-OCT, increases in nasal and temporal iridocorneal angles were significant at the post-operative evaluations ${ }^{16}$ whereas with iris-fixated IOLs (and using the Pentacam) the angles became smaller following sur- 
gery ${ }^{17}$. Thus types of ophthalmic surgery can have important influences of ACA and methods to identify such variation such as UBM, AS-OCT and SP are naturally useful in clinical and research contexts.

The purpose of this study was to investigate shortterm intra-ocular variability of the ocular ACA in different meridians of the anterior segment of a few eyes using Scheimpflug photography. This is perhaps pertinent given the apparent scarcity of information or papers on the topic. A useful method for representing such variation using polar plots is also provided here (possibly for the first time in relation to ACA) that potentially could assist with better understanding of variation in ACA. Polar profiles have previously been used for representation of other parameters in optometry and vision science, for example, by van Gool and Harris for variances of dioptric power ${ }^{18-19}$.

\section{Methods}

The study proposal satisfied the requirements of the Declaration of Helsinki and was approved according to the required process of the University of Johannesburg. After signing the applicable informed consent document, each subject also completed a simple, mainly biographical questionnaire that also included some questions on general and ocular health relevant to the purposes of this study. Various standard procedures such as autorefraction and non-contact tonometry were performed and thereafter an Oculus Pentacam was used to repeatedly measure the right eyes only of three female Caucasoid subjects, all 22 years of age. Ambient luminance was similar for all three subjects and measurements were obtained in photopic conditions. Each eye was measured ten times in rapid succession, thus resulting in 10 maps per eye. (The time required to obtain the 10 maps for each subject was about 15-20 minutes although the instrument measures a single map in less than two seconds. Much of the period involved in obtaining multiple maps relates to the time needed to obtain proper alignment of the pupil centre via the instrument's focusing and alignment systems.) Each topographic map included 25 meridional or cross-sectional scans of the anterior segment of the eye concerned. During each measurement of a map, the subject kept both eyes open while observing the internal instrument target with their right eye. Between measurements subjects removed their heads from the instrument's head and chin rests. One person obtained all measurements for the three subjects and the instrument was refocused and realigned between each measurement in the series of 10 per eye. This particular approach encourages greater independence of measurements but also can produce greater variability in measurements. No attempt was made to control head position, for example, with bite bars. Drugs such as mydriatics or cycloplegics were not used and thus the eyes were in their natural states and this study was based upon a possibly worse-case scenario where variation in the parameter of interest, namely anterior chamber angle would be maximal.

\section{Statistical analysis}

Measurements for the three eyes were analysed with software, namely Statistica and Matlab. The parameter of interest was the ocular anterior chamber angle (units, degrees) and 50 values (from both ends of each of 25 meridional scans) were obtained from each map and so for a single subject a 10 x 50 matrix of values for ACA for the various meridians was obtained. Within each subject, the short-term variation of the angles for the meridians involved was investigated with box and whisker plots of medians and quartile ranges and other line and polar plots ${ }^{18,19}$ were also used. Friedman analysis of variance (ANOVA) ${ }^{20}$ was used separately for each individual to determine whether there was any significant statistical difference in medians (for ACA) across the meridians of each eye concerned.

\section{Results}

In Figure 1 box and whisker plots of medians (the small squares) and quartile ranges (larger rectangles or boxes) are used to graphically represent the variation in ACA for the various meridians of right eyes of three young, female subjects. Maxima and minima for the different meridians are also provided using whiskers. Medians have been used in Figure 1 since the data for different meridians possibly departs from univariate normality and thus a non-parametric approach (rather than a parametric one with, say, means and standard deviations) was preferred. (But later in this paper both the meridional means and medians will be considered briefly.) Figure 1(a) is for Subject 1 while parts (b) and (c) of the figure are for Subjects 
2 and 3 respectively. In each part of Figure 1, the $x$ axis represents change or variation in the reference meridian while the $y$-axis represents the magnitude in degrees for the ocular ACA. The $x$-axis begins by representing the superior nasal quadrant (meridians $\left.0-90^{\circ}\right)$ and then the superior temporal quadrant $(90-$ $\left.180^{\circ}\right)$. Thereafter, from 180 to $360^{\circ}$ the inferior temporal and inferior nasal quadrants are indicated. Thus in Figure 1(a) we note that initially the angle decreases in size in the superior nasal quadrant of this right eye and then it increases in magnitude from about 100 to $180^{\circ}$, that is, as we travel from the superior part of the angle towards the temporal horizontal part of the angle. Then after a maximum at about 200 degrees the angle again decreases in size (in the inferior temporal quadrant) but remains larger than that found at, say, $100^{\circ}$. Then in the inferior nasal aspect the angle increases towards the horizontal meridian. (In Table 1 the mean, rather than median, angles follow a very similar pattern.) In Figure 1(a) we find the curve formed is roughly sinusoidal in appearance with the anterior chamber angle slightly larger than $20^{\circ}$ near the vertical meridian but almost $40^{\circ}$ in the inferior quadrant near semi-meridian $270^{\circ}$. The largest angles $\left(>50^{\circ}\right)$ for this subject are located near the horizontal meridian. Figure 1(a) also suggests that the variation in medians for the different meridians of the eye is quite profound and the medians range from about 22 to $54^{\circ}$ dependent upon meridian involved. Similar, and roughly sinusoidal, curves are noticeable for the other two subjects also in Figures 1(b) and 1(c).

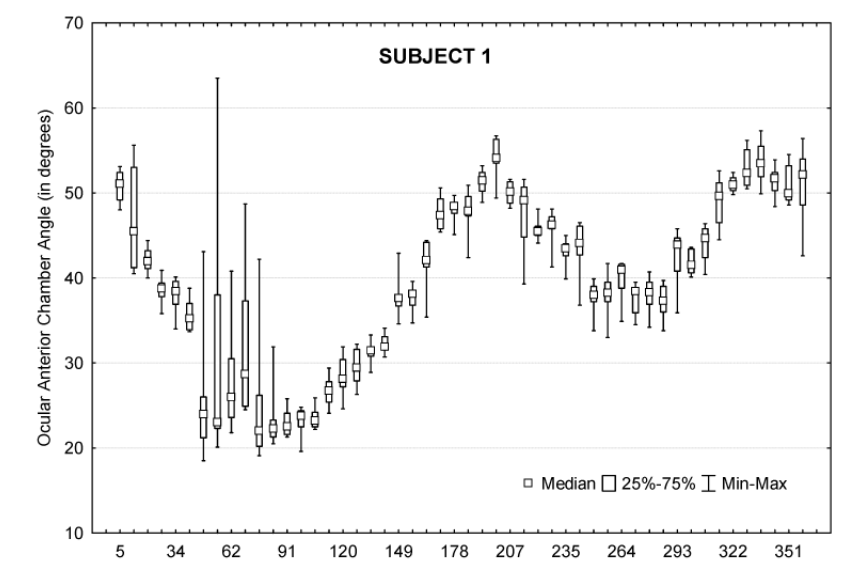

a)

Meridian (in degrees)
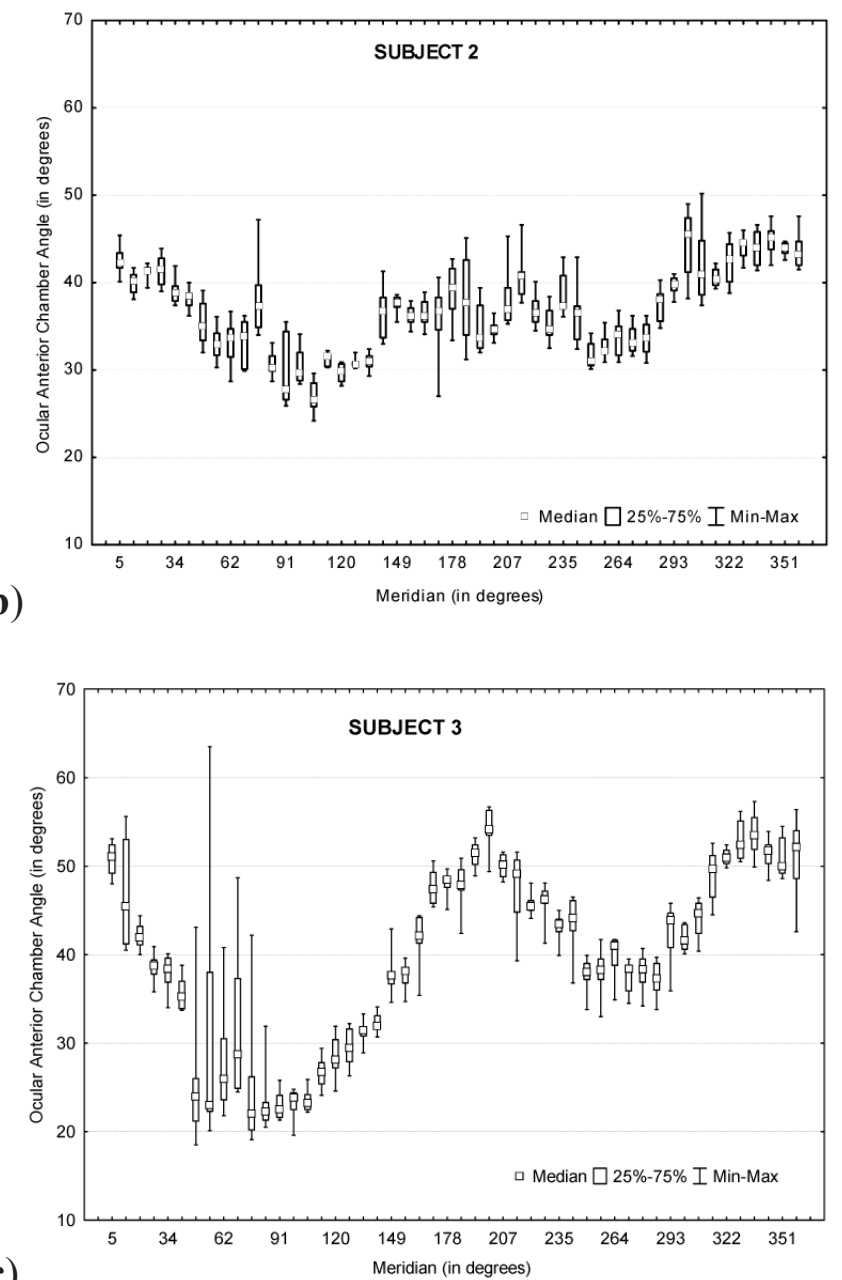

c)

Figure 1. Box and whisker plots of the medians (small boxes) and quartile ranges (larger rectangles) for the ocular anterior chamber angles (ACA) for the different meridians of the right eyes of three young, female subjects. All measurements of ACA were obtained using the Oculus Pentacam repeatedly within each subject ( $N=10$ maps per right eye) over a short time period. Maxima and minima for individual meridians are also indicated using the whiskers and possible outliers are present for a few meridians, such as near $60^{\circ}$ in Subjects 1 and 3. But, excluding these few meridians where outliers were possibly relevant, the within-meridian or intra-meridional variances in ACA are relatively small for each subject. That is, each quartile range (also called the inter-quartile range $\left(Q_{1}\right.$ and $\left.Q_{3}\right)$ and essentially the width of the range about the median concerned including 50\% of the cases) was mostly small. a) Subject 1, b) Subject 2 and c) Subject 3 .

Figure 2 allows for easier comparison of ACA for the different subjects. In this figure six coloured curves are used to provide the results for both means (solid lines) and medians (dashed) for the various meridians for the three eyes concerned. Results for ACA for Subject 1 are shown using red while results for 
subjects 2 and 3 are shown using blue and green respectively. For most meridians the means (see Table 1 for these means) and medians are not too different but there are a few regions where the solid and dashed lines are seen to be somewhat different (largely relating to the presence of isolated outliers and here the medians are probably more appropriate measures of central tendency, although possible outliers could be removed and then the means and medians for the regions or meridians concerned would likely become more similar).

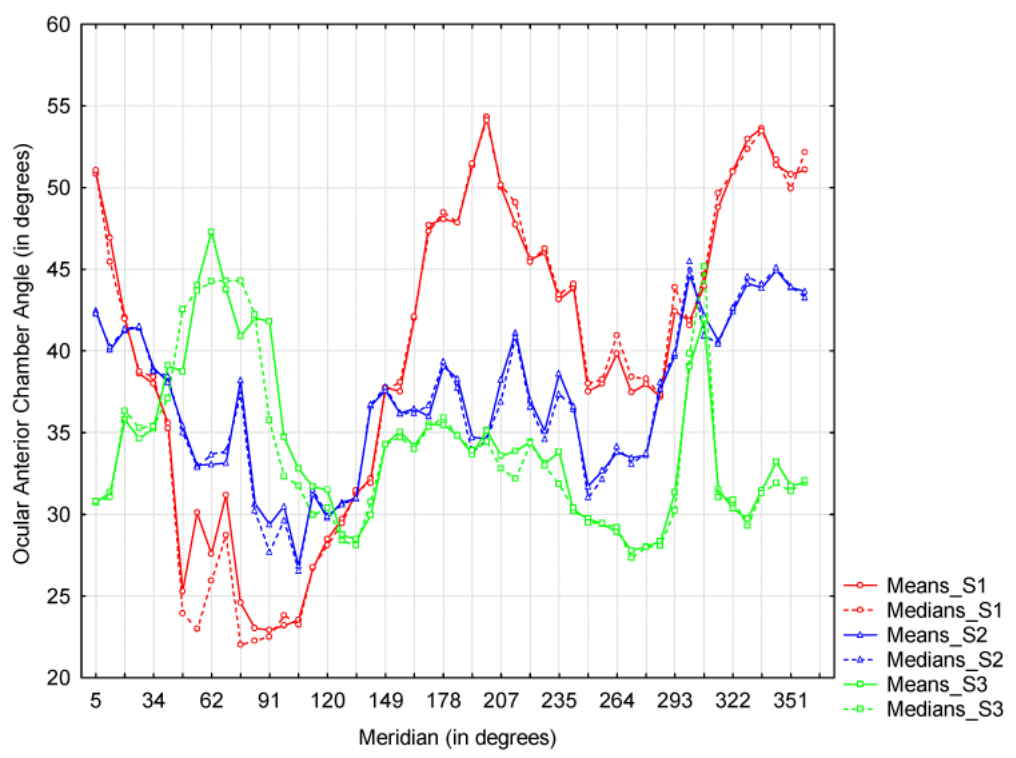

Figure 2. Variation in the means and medians for ACA for different meridians of three young female eyes are indicated. For each eye, and subject, solid or dashed coloured curves represent the means and medians respectively. Results for Subject 1 are in red, Subject 2 in blue and Subject 3 in green. Possible outliers in Subjects 1 and 3 (near meridian $60^{\circ}$ ) result in the means and medians becoming a little different and the solid and dashed lines are seen to depart from one another but mostly within specific meridians the intra-subjective means and medians are similar.

Table 1. Means and standard deviations for meridians 5 to $358^{\circ}$ are provided for the ocular anterior chamber angle for the right eyes of three young female subjects, all aged 22 years. (Each of the means in columns two to four is the average of 10 measurements as obtained using the Oculus Pentacam.) The units are degrees throughout and the usual mathematical convention applies (that is, angles are measured counter-clockwise from the horizontal meridian). Thus the superior-nasal quadrant of the right eye would be represented by meridians 5 to $84^{\circ}$. Since Figure 1 suggests for the different subjects that for most meridians the means and medians were similar, the parametric means are provided here rather than the medians.

\begin{tabular}{|c|c|c|c|}
\hline Meridian & Subject 1 & Subject 2 & Subject 3 \\
\hline 5 & $50.85 \pm 1.82$ & $42.45 \pm 1.46$ & $30.74 \pm 1.35$ \\
\hline 12 & $46.90 \pm 5.86$ & $40.07 \pm 1.24$ & $31.32 \pm 1.26$ \\
\hline 19 & $42.05 \pm 1.42$ & $41.27 \pm 0.83$ & $35.81 \pm 2.13$ \\
\hline 27 & $38.60 \pm 1.47$ & $41.45 \pm 1.66$ & $34.64 \pm 2.44$ \\
\hline 34 & $37.99 \pm 1.89$ & $38.96 \pm 1.32$ & $35.26 \pm 1.35$ \\
\hline 41 & $35.57 \pm 1.83$ & $38.09 \pm 1.12$ & $39.10 \pm 7.81$ \\
\hline 48 & $25.28 \pm 6.87$ & $35.41 \pm 2.35$ & $38.74 \pm 6.58$ \\
\hline 55 & $30.11 \pm 13.54$ & $33.01 \pm 1.75$ & $44.00 \pm 0.90$ \\
\hline 62 & $27.58 \pm 5.84$ & $33.06 \pm 2.50$ & $47.30 \pm 9.17$ \\
\hline 70 & $31.19 \pm 8.03$ & $33.14 \pm 2.54$ & $43.76 \pm 2.20$ \\
\hline 77 & $24.57 \pm 6.91$ & $38.19 \pm 4.06$ & $40.90 \pm 6.58$ \\
\hline 84 & $23.02 \pm 3.28$ & $30.65 \pm 1.30$ & $42.01 \pm 7.22$ \\
\hline 91 & $22.91 \pm 1.50$ & $29.38 \pm 3.84$ & $41.80 \pm 17.70$ \\
\hline 98 & $23.19 \pm 1.64$ & $30.49 \pm 1.95$ & $34.72 \pm 6.25$ \\
\hline 106 & $23.53 \pm 1.23$ & $26.85 \pm 1.79$ & $32.80 \pm 5.25$ \\
\hline 113 & $26.70 \pm 1.80$ & $31.27 \pm 0.68$ & $31.69 \pm 5.93$ \\
\hline 120 & $28.47 \pm 2.15$ & $29.78 \pm 0.99$ & $31.49 \pm 3.21$ \\
\hline 127 & $29.70 \pm 2.07$ & $30.74 \pm 0.51$ & $28.75 \pm 2.23$ \\
\hline 134 & $31.26 \pm 1.29$ & $30.98 \pm 0.94$ & $28.45 \pm 1.31$ \\
\hline 142 & $32.21 \pm 1.03$ & $36.64 \pm 2.64$ & $29.92 \pm 2.01$ \\
\hline 149 & $37.76 \pm 2.11$ & $37.59 \pm 0.91$ & $34.30 \pm 2.15$ \\
\hline 156 & $37.53 \pm 1.54$ & $36.19 \pm 1.09$ & $35.07 \pm 1.20$ \\
\hline 163 & $42.00 \pm 2.70$ & $36.46 \pm 1.62$ & $34.16 \pm 1.63$ \\
\hline 171 & $47.71 \pm 1.90$ & $35.99 \pm 3.68$ & $35.51 \pm 1.60$ \\
\hline 178 & $48.07 \pm 1.34$ & $39.08 \pm 2.92$ & $35.47 \pm 1.90$ \\
\hline 185 & $47.86 \pm 2.37$ & $38.28 \pm 4.90$ & $34.83 \pm 2.29$ \\
\hline 192 & $51.32 \pm 1.35$ & $34.73 \pm 2.70$ & $33.67 \pm 0.76$ \\
\hline 199 & $54.33 \pm 2.18$ & $34.61 \pm 0.99$ & $35.13 \pm 3.71$ \\
\hline 207 & $50.03 \pm 1.34$ & $38.25 \pm 3.55$ & $33.59 \pm 4.14$ \\
\hline 214 & $47.74 \pm 3.95$ & $41.13 \pm 2.88$ & $33.85 \pm 4.15$ \\
\hline 221 & $45.59 \pm 1.16$ & $36.98 \pm 1.87$ & $34.43 \pm 4.41$ \\
\hline 228 & $46.00 \pm 1.96$ & $35.10 \pm 1.85$ & $33.09 \pm 1.98$ \\
\hline 235 & $43.15 \pm 1.39$ & $38.63 \pm 2.38$ & $33.81 \pm 4.88$ \\
\hline 242 & $43.84 \pm 2.88$ & $36.45 \pm 3.29$ & $30.18 \pm 1.89$ \\
\hline 250 & $37.53 \pm 1.92$ & $31.72 \pm 1.57$ & $29.74 \pm 1.40$ \\
\hline 257 & $38.00 \pm 2.48$ & $32.67 \pm 1.40$ & $29.40 \pm 0.79$ \\
\hline 264 & $39.85 \pm 2.35$ & $33.81 \pm 2.07$ & $28.92 \pm 0.85$ \\
\hline 271 & $37.46 \pm 1.86$ & $33.47 \pm 1.55$ & $27.79 \pm 2.05$ \\
\hline 278 & $37.96 \pm 2.17$ & $33.61 \pm 1.89$ & $28.01 \pm 1.33$ \\
\hline 286 & $37.17 \pm 1.83$ & $37.66 \pm 1.82$ & $28.35 \pm 1.50$ \\
\hline 293 & $42.43 \pm 3.52$ & $39.73 \pm 1.00$ & $31.32 \pm 2.74$ \\
\hline 300 & $41.85 \pm 1.36$ & $44.68 \pm 3.57$ & $39.05 \pm 5.81$ \\
\hline 307 & $43.97 \pm 2.13$ & $42.19 \pm 4.54$ & $41.91 \pm 8.07$ \\
\hline 314 & $48.80 \pm 2.78$ & $40.61 \pm 1.05$ & $31.51 \pm 1.83$ \\
\hline 322 & $51.02 \pm 0.88$ & $42.44 \pm 2.43$ & $30.36 \pm 2.10$ \\
\hline 329 & $52.97 \pm 2.12$ & $44.14 \pm 1.34$ & $29.73 \pm 1.85$ \\
\hline 336 & $53.64 \pm 2.44$ & $43.87 \pm 2.05$ & $31.47 \pm 1.02$ \\
\hline 343 & $51.38 \pm 1.68$ & $44.94 \pm 1.74$ & $33.21 \pm 3.69$ \\
\hline 351 & $50.80 \pm 2.14$ & $43.88 \pm 0.71$ & $31.75 \pm 1.20$ \\
\hline 358 & $51.08 \pm 4.06$ & $43.64 \pm 1.87$ & $31.94 \pm 1.25$ \\
\hline
\end{tabular}


Polar plots (see Figure 3) possibly enhance interpretation of both intra- and inter-meridian variation in ACA for the different subjects. In each part of Figure 3 , polar profiles (dashed black lines) represent results for ACA from the 10 successive Pentacam maps for a particular eye. Two polar profiles (red solid lines) in each part of Figure 3 represent intra- and inter-meridian variation in the means and medians. These red profiles are essentially the same as in Figure 2 but interpretation is perhaps easier since we use the polar plot as if we were looking directly at a right eye. That is, the upper part of the polar plot represents the superior part of the eye while the lower part includes results for the inferior angles.

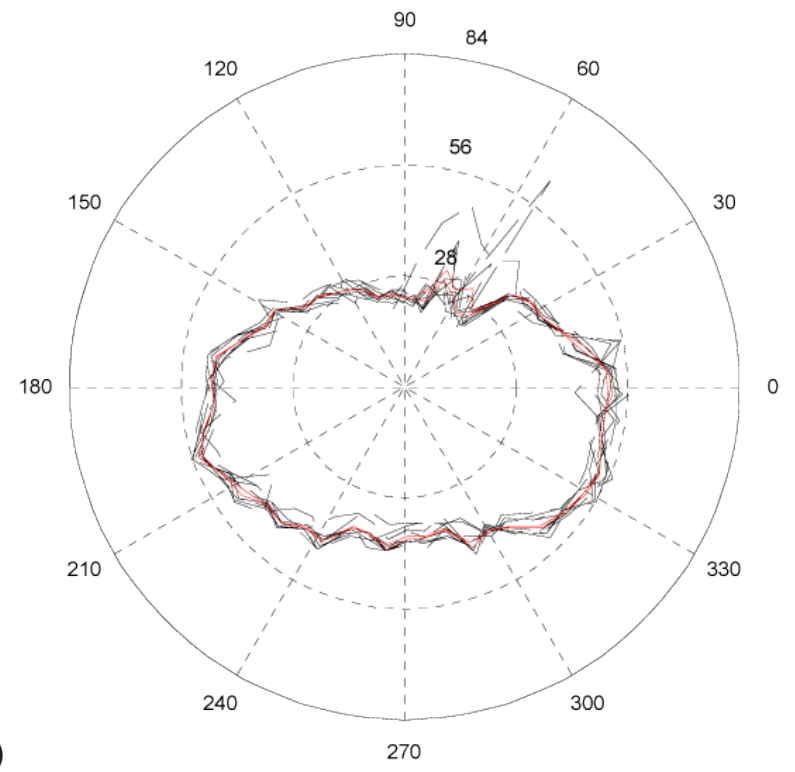

a)

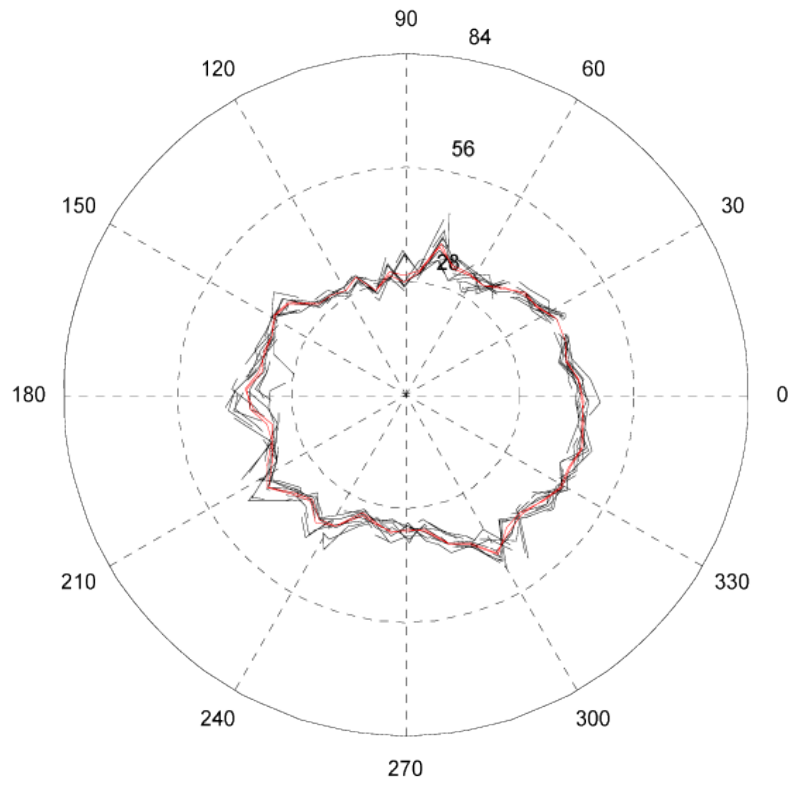

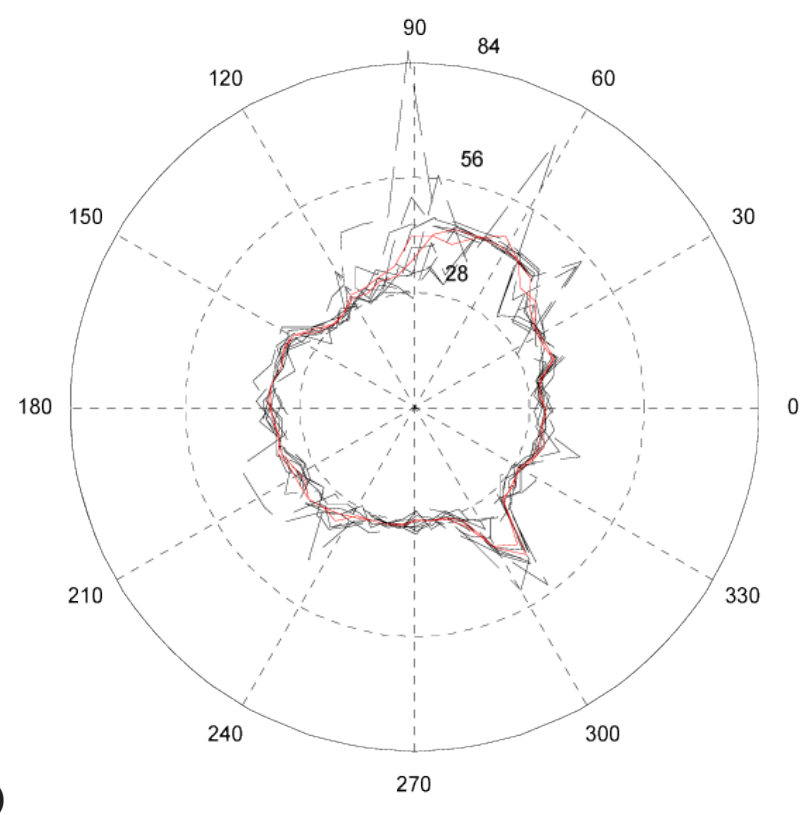

Figure 3. Polar plots for the raw data for ocular ACA for all meridians and the resulting means and medians for the measured meridians for three subjects are provided. For each subject, there are 10 polar profiles (the dashed black profiles) that represent meridional results from 10 consecutive maps determined using the Oculus Pentacam. An additional two red solid profiles for each subject then represents the meridional means and medians respectively as determined from the raw data. a) Subject 1, b) Subject 2 and c) Subject 3 .

The nasal quadrants are on the right side of the polar plot (exactly as when looking directly at the subject). If the ACA were uniform or constant, that is, invariant with respect to change of reference meridian then circles of constant radius would result.

In Figure 4 the means (the black polar profiles) for the different meridians for Subjects 1 to 3 are plotted again together with other polar profiles (in red) that define the $95 \%$ confidence regions on the means. The blue polar profiles represent the maxima and minima for the different meridians. These polar plots are very useful in establishing the extent to which the Oculus Pentacam is providing repeatable results both within and across the different meridians. For example, in Subject 1 (Figure 4(a)) except for a few meridians in the superior-nasal quadrant near $55^{\circ}$ the five polar profiles are relatively similar. If we were to remove a few outliers from the raw data used to calculate the means and other statistics then even in the superiornasal quadrant we would find that the instrument is mostly providing similar and thus repeatable results. The polar plot (Figure 4(b)) for Subject 2 further sup- 
ports this latter finding but in Subject 3 we can see that the measurements are less repeatable (profiles are further apart) in some meridians. In all instances removal of a limited number of possible outliers would result in the measurements becoming more repeatable and thus the instrument is capable of providing repeatable results for ACA, at least over the short-term and given the potential limitation that only three eyes are included here. Polar plots can also be used to plot other statistics such as profiles of skewness and kurtosis for the different meridians, profiles for the medians and lower $\left(Q_{1}\right)$ and upper $\left(Q_{3}\right)$ quartiles, or profiles for the quartile deviations (essentially the semi-interquartile range or one-half of the quartile range or $\left.\frac{1}{2}\left(Q_{3}-Q_{1}\right)\right)$ for the various meridians. Quartile deviation, sometimes called the median absolute deviation (MAD), is less affected by outliers and is a robust measure of dispersion in comparison with standard deviation. Polar plots, as in Figure 4, thus allow for a very detailed and in-depth analysis of ACA within individual eyes and could similarly be applied across many eyes instead.

a)

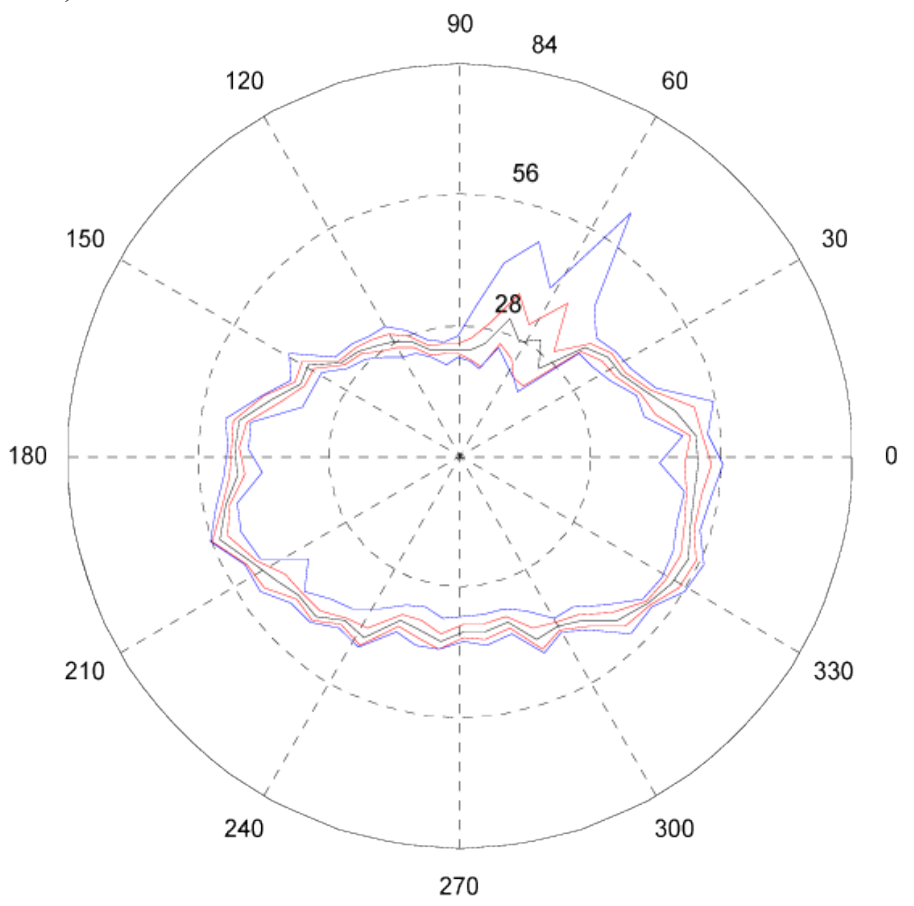

b)

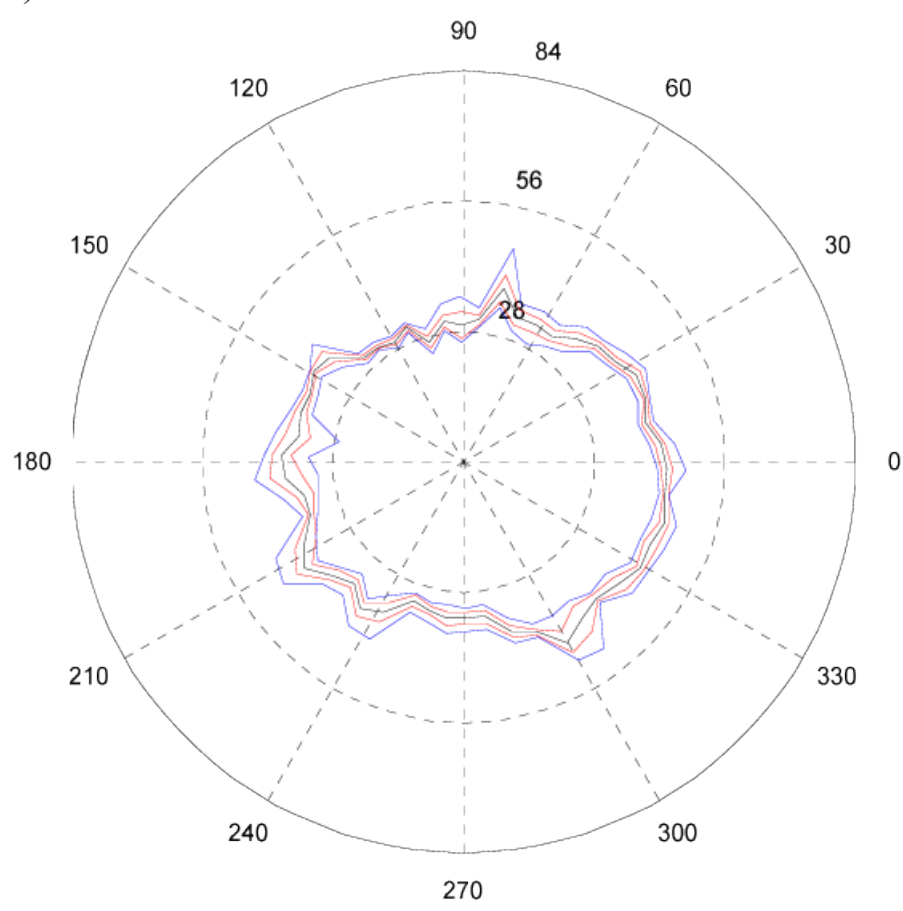

c)

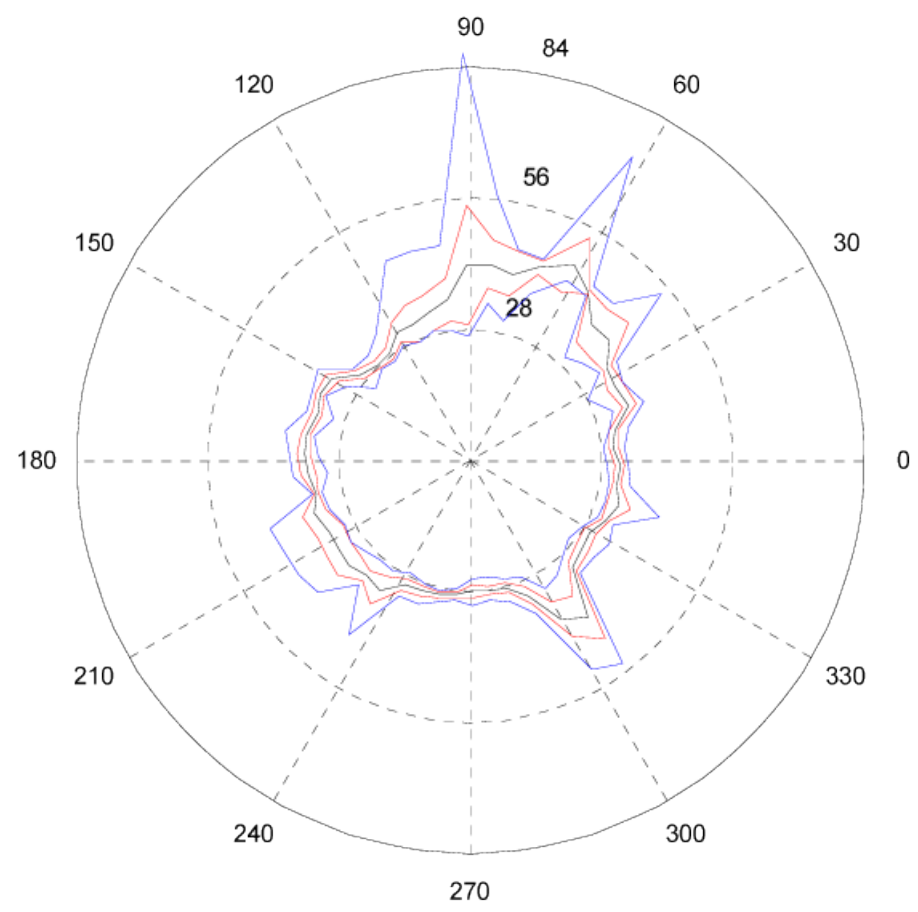

Figure 4. Polar plots of intra- and inter-meridian ACA means and $95 \%$ confidence regions on these means for each of the three subjects. In each part of this figure the black polar profile represents the mean ACA for each of the different meridians while the red profiles are used to indicate the upper and lower limits for the $95 \%$ confidence regions on these means. The blue polar profiles indicate maxima and minima for all meridians. a) Subject 1, b) Subject 2 and c) Subject 3 . 
Figure 5, where the means and medians using polar coordinates are plotted for the three subjects, makes it easy to interpret and compare results for the different eyes. For instance, we can see that Subject 1 (the red polar profiles) had the smallest angles of the three subjects in the superior quadrants but had the largest angles otherwise of the three subjects except near $300^{\circ}$. Except for some meridians of the superior quadrants, subject 3 (green profiles) had the smallest anterior chamber angles of these three subjects.
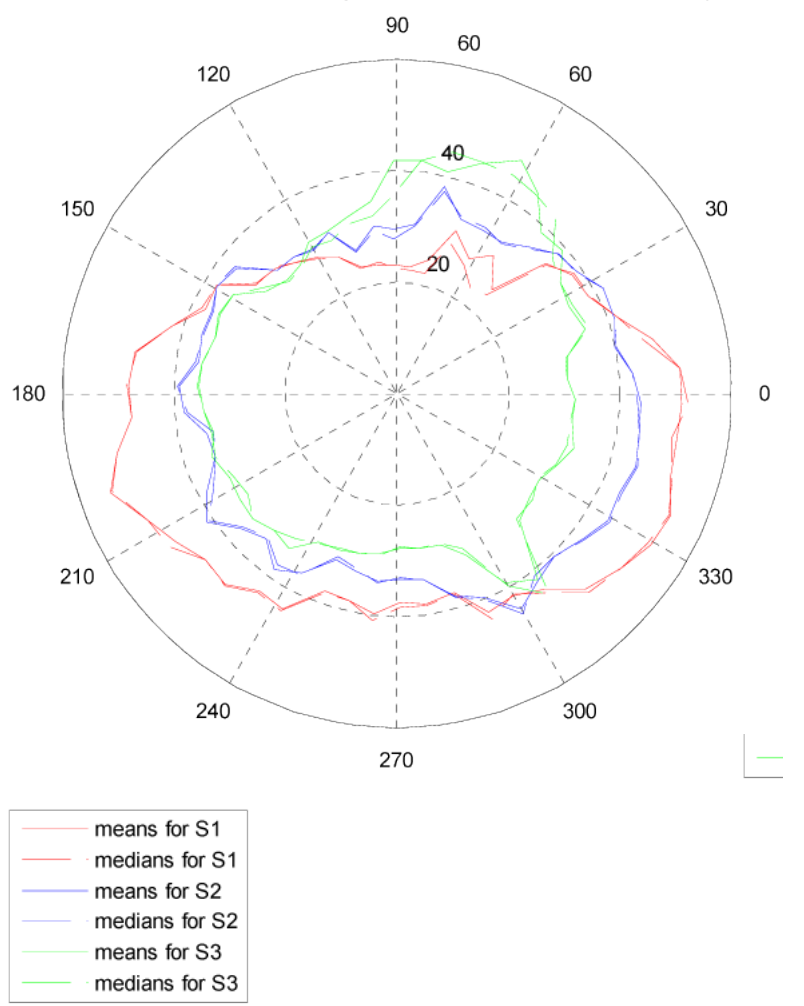

Figure 5. Polar plots of the meridional means (solid profiles) and medians (dashed profiles) for ocular ACA for the right eyes of three young female subjects. Different colours are used to represent the different subjects (see the legend). The radial coordinates range from 0 to $60^{\circ}$, that is, the further away a coloured profile is from the polar origin the larger the ACA for the meridian/s concerned. Numbers around the circumference represent reference meridian in degrees. The superior nasal quadrants for these right eyes are represented by meridians $0-90^{\circ}$.

Polar profiles are again used in Figure 6 for each of the three eyes concerned but here the profiles represent change in a chosen measure of variability, namely that of standard deviation for the different meridians. The red profile is for Subject 1 while blue and green are used for the meridional standard deviations for Subjects 2 and 3 respectively. Should the standard deviations for different meridians for an eye be the same, then a circle of constant radius would be found.
We can see, however, that within each eye or subject that the standard deviations vary across the meridians and this is particularly noticeable where possible outliers were present (for example, for Subject 1 near the meridian of $55^{\circ}$ (see the red polar profile) or near the vertical meridian for subject 3 as indicated with the green profile instead). Removal of possible outliers would change these polar maxima but here such outliers were not removed from the data since samples were relatively small at only 10 observations or measures per meridian per subject. Although not included here, it would also be possible to use similar polar plots for MAD or quartile deviation (a nonparametric measure of variation) for each subject. In this paper the means and medians within subjects were relatively similar (with the possible exception of one or two meridians) and thus parametric means and standard deviations (as in Figure 6) were used for the analysis of variability within and across the different meridians.

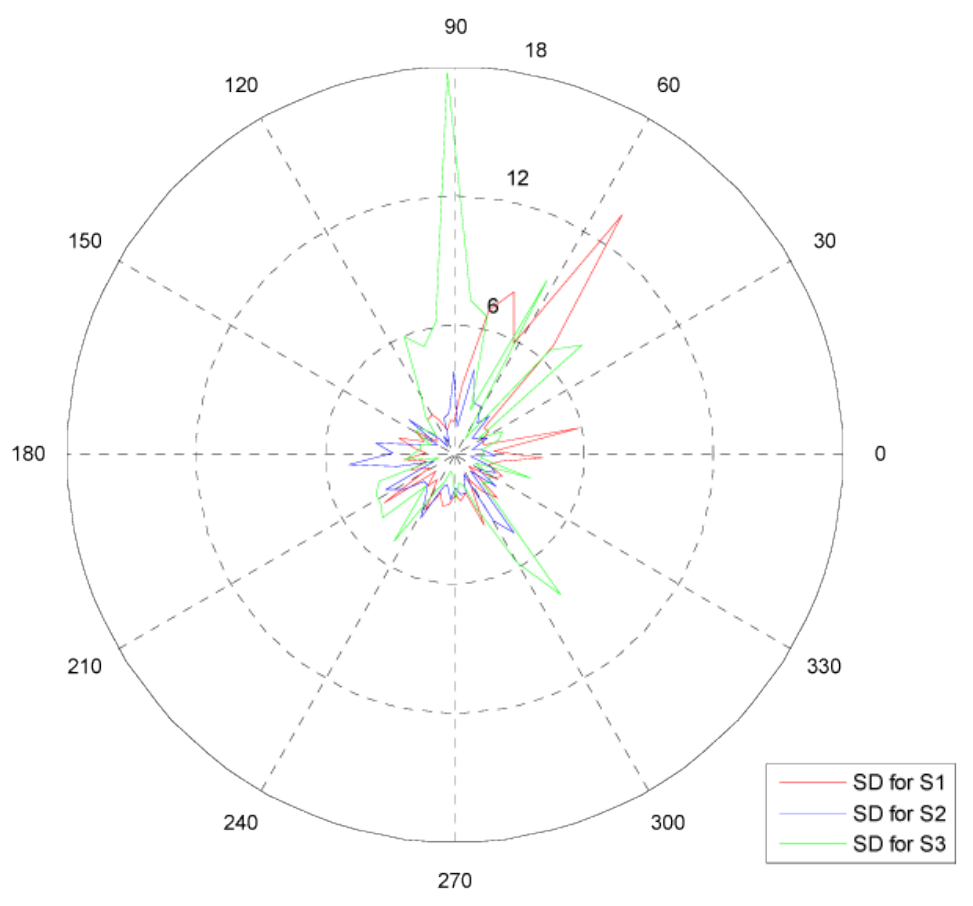

Figure 6. Polar profiles are given for Standard Deviations (SD; units are degrees) for the ACA data for the different meridians of the right eyes of the three subjects involved. Different colours are used to represent the different subjects (see legend). Note that the blue polar profile is mostly close to the polar origin and within the first black, dashed-line, circle and thus for this subject (number 2) the standard deviations for the different meridians were relatively small and less than $4.5^{\circ}$. The same is largely true for Subject 3 (green) with the exception of meridians near 48, 62, 91 and $307^{\circ}$. The standard deviations for the different meridians for Subject 1 (the red profile) are also mostly near the polar origin with the exception of meridians near 12, 55 and $70^{\circ}$. If a few outliers were removed these profiles would probably be found closer to the polar origin. 
Table 2. Means for ocular ACA, in degrees, for the different quadrants for the right eyes of the three subjects are indicated.

\begin{tabular}{|l|l|l|l|l|}
\cline { 2 - 5 } \multicolumn{1}{c|}{} & $\begin{array}{l}\text { Superior-Nasal } \\
\text { Quadrant }\end{array}$ & $\begin{array}{l}\text { Superior-Temporal } \\
\text { Quadrant }\end{array}$ & $\begin{array}{l}\text { Inferior-Temporal } \\
\text { Quadrant }\end{array}$ & $\begin{array}{l}\text { Inferior Nasal } \\
\text { Quadrant }\end{array}$ \\
\hline Subject 1 & 34.48 & 33.16 & 45.44 & 46.19 \\
Subject 2 & 37.15 & 33.19 & 36.03 & 41.14 \\
Subject 3 & 38.63 & 33.39 & 32.55 & 32.03 \\
\hline
\end{tabular}

Friedman analysis of variance ${ }^{20}$ (ANOVA) was used for each subject separately to determine whether or not there were significant differences in the medians for the different meridians. The Friedman ANOVA is a non-parametric test where the null hypothesis is that measures of ACA for the variables (here the various meridians) are drawn from the same populations, that is, the medians for the different meridians are identical. For each of the three subjects concerned, the medians within each subject were found to differ significantly across the meridians of the eye involved. This was not unexpected due to anatomical factors relating to ACA and confirmed our visual inspection of the medians in Figures 1 to 3 .

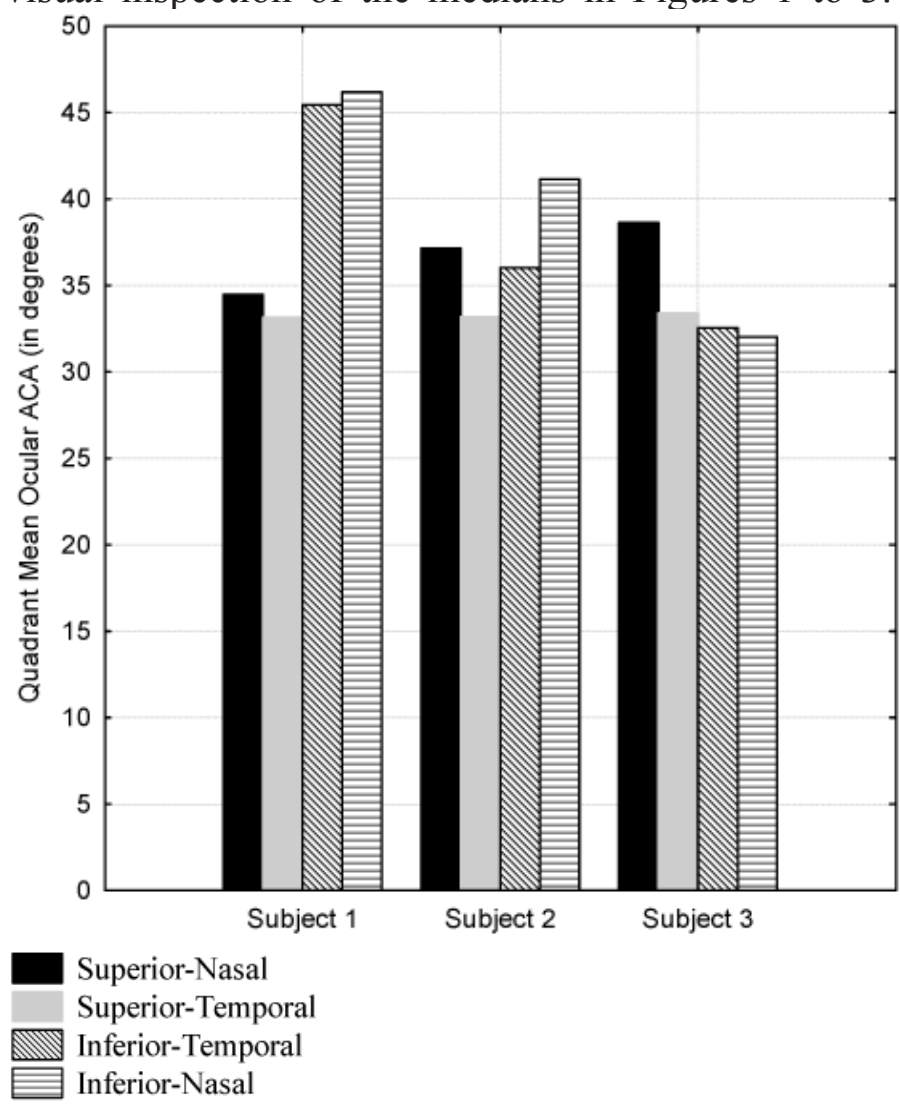

Figure 7. Mean ocular anterior chamber angles for the four quadrants for the right eyes of three young, female subjects are indicated. Different bars (see legend) represent the various quadrants and, for example, black is used for the superior-nasal quadrant. The means for this figure can be found in Table 2.
Table 2 and Figure 7 include the mean ACA for each quadrant of the three right eyes concerned. The means were determined by averaging the relevant meridional means from Table 1. For example, for the superior-nasal quadrant of, say, Subject 1 an overall quadrant mean was determined from the individual means for the meridians from 5 to $84^{\circ}$ and, similarly, for other quadrants and subjects. The quadrant means for the three eyes of the subjects are provided in Table 2. In Subject 1, for example, we can see from Figure 7 that the mean ACA was greater in the two inferior quadrants $\left(45.44\right.$ and $\left.46.19^{\circ}\right)$ while the superior-temporal quadrant (grey bar) was the narrowest with a mean of $33.16^{0}$. Of the three subjects, Subject 3 had the largest angle (mean) for the superior-nasal quadrant (compare the three black bars in Figure 7).

\section{Discussion and Conclusion}

This paper provides an important contribution in that there is apparently little in the way of quantitative information or studies available concerning the use of Scheimpflug photography for comprehensive and repeated measurement of the ocular anterior chamber angle, and especially across the different meridians of the eye. Most studies of ocular ACA have mainly used ultrasound biomicroscopy or optical coherence tomography or other approaches that may be more subjective in nature. A limited number of studies have suggested that the repeatability of measures of the ACA with SP might not be that good and thus this paper investigates this topic and is partly intended to either confirm or disaffirm this particular finding. As such a decision was made to measure only a few eyes but in great detail and specifically obtain repeated measurements for the different meridians of those eyes. Thus the major emphasis was more towards an intra-subjective study design rather than the intersubjective one mostly used in other studies. The polar approach to representation of the ACA also assists towards easier analysis of the data collected within 
each eye. (See references 18 and 19 for polar plots that previously were used by van Gool and Harris for representation and quantification of a different variable, namely variance of dioptric power.)

Although only three eyes were included in this study each was measured repeatedly and this allowed for the effective determination of quantities such as mean and median ACA for the different meridians. Short-term variation of ACA in the various meridians thus could be investigated and for most meridians, if a limited number of outliers are ignored, both the standard deviations and quartile deviations were relatively small having magnitudes of only a few degrees. However, the variation within subjects but across meridians could be quite pronounced (see Figure 1, for example). Further investigation in larger samples of eyes and also in relation to conditions such as narrow or angle closure glaucoma would be useful. Another aspect of this study is that all three subjects were young (22 years of age) and female and future work should also include males and also extend the age range involved since various studies ${ }^{2,6,12}$ have found that ACA is influenced by both age and gender. The race of the subjects in this study was limited to Caucasoid and further work with subjects of other races would also be useful.

Meridional plots of median ACA and related quartile ranges $\left(Q_{3}-Q_{1}\right.$ or the $75^{\text {th }}$ percentile minus the $25^{\text {th }}$ percentile) as in Figure 1 are useful in that they allow one to study and quantify intra-subjective variation in ACA both within particular meridians as well as across the different meridians. (Parametric plots of mean ACA and standard deviations could be used instead but since the number of observations (at 10) per meridian was relatively small the non-parametric method was initially preferred, and this method also makes no assumptions about normality of the data. The polar plots (of Figures 3 and 4) similarly are also very informative about short-term intra-subjective variation of ACA and are perhaps particularly useful as they provide an holistic and intuitive method of rapidly assessing the nature and magnitude of variation present. The author is unaware of other plots of this type being previously used to represent and investigate variation of ACA although they have been used before in other contexts in optometry ${ }^{18,19}$. Such plots can also be used to indicate inter-subjective (see Figure 5 for example) rather than intra-subjective var- iation of ACA and thus polar profiles for larger samples of eyes, say 50 eyes with chronic narrow angle glaucoma, could be represented upon a single polar plot and thereby compared with relative ease. Also, outliers and other departures from univariate normality could be identified and investigated using such plots; for example, in Figure 3(a) one might compare the median profile (in red) to the raw data (10 profiles in black) and the outliers in the meridians near $55^{0}$ suggest the likelihood of positive skewing in the data for those meridians. (Thus we are considering the pattern or arrangement of the black profiles with respect to the polar profile of medians.)

This study was in the nature of a preliminary but detailed study of a few eyes only but some indications are also present that the meridional means and medians are not too different (for example, compare the profiles for means and medians in Figure 5) in the absence of possible outliers and thus perhaps mainly parametric statistics and methods can be applied and this was done in some parts of this paper. But further investigation in a larger number of eyes (and possibly with more than 10 maps per eye) is needed to confirm this preliminary indication. The issue of univariate normality or otherwise of ACA has not been extensively studied or emphasised in this particular paper but is briefly mentioned in places.

One of the possible difficulties with the measurement of ACA with the Pentacam (but also with alternative approaches such as UBM or AS-OCT) is that cursors, callipers or other measuring objects are positioned or aligned with structures in the anterior chamber angle. This positioning may be subjectively and manually performed by the instrument operator, or software in the instrument may automatically place the measuring objects or devices in their initial state, with perhaps some subjective re-adjustment allowed by the user. Thus there is room for some uncertainty or misalignment that could possibly influence findings to some extent. With specifically Scheimpflug photography of multiple meridians care must be exercised to insure that subjects or patients keep their eyes properly open during acquisition of data, otherwise the eyelids may interfere with effective measurement and may distort the digital images for some meridians so that measuring ACA in said meridians could become problematic and difficult. Automated image processing software significantly reduces or 
eliminates the subjective nature of placing these callipers and, for example, Leung et al describe such an algorithm for ultrasound biomicroscopy that produces repeatable and fully automated measurements of angle opening distances and trabecular-iris angles ${ }^{21}$. But the same algorithm could be applied to SP and the Pentacam already has such automated methods included for measurement of ACA.

Another concern with using Scheimpflug photography to measure ACA is that the limbal or corneoscleral junction can sometimes be overexposed (due to the scleral tissue being very white in comparison to the cornea and anterior chamber itself) possibly making it difficult in some meridians to define the actual apex of the anterior chamber angle itself. This was found also to be true for images acquired in this study but the automated software in the instrument appeared able to use extrapolation to locate the angles concerned and provide measures of ACA. But, some have argued that in comparison with other methods such as AS-OCT that the possible inaccuracies due to this issue could be large enough to create problems with, for example, proper evaluation of angle-supported implants after cataract extraction ${ }^{22,23}$.

The measures for mean quadrant ACA (as provided in Table 2 and Figure 7 of this study) are not dissimilar to what might be expected for young, female healthy eyes or to that cited in the literature (see below). Most of these measures for the different quadrants involved were between $30-40^{\circ}$ with the angles in the inferior quadrants a little larger in two of the three subjects concerned. The apparent scarcity of similar studies in the scientific literature to this particular study made it difficult to compare or contrast results to those by others. Previous researchers do not appear to have investigated short-term variation of ACA extensively and neither have they studied meridional variation of ACA to any major degree. Where they have investigated the anterior chamber they appear to have mainly used other methods such as ultrasound biomicroscopy or optical coherence tomography rather than Scheimpflug photography (perhaps partly relating to possible concerns ${ }^{2,9}$ with less satisfactory repeatability with instruments such as the Pentacam when measuring quantities such as ACA through SP; there appears to be better repeatability when using the instrument to measure other parameters $9-10,13,25$ such as central corneal thickness $2,10,11,13,14,26$ or vol- umes ${ }^{2,12}$ ) and thus an attempt will be made to briefly compare some of the findings in this paper to such studies despite the obvious limitation in terms of the differences in technology or instrumentation used to perform the research and measure ACA. The results for mean ACA with SP in this study (see Table 2 and Figure 7) for the different quadrants were not too dissimilar to that of Xu et $a l^{6}$ although they only measured the nasal and temporal quadrants with AS-OCT and they used an inter-subjective study design with almost 3000 Chinese subjects of an older age than applicable for this study. Wirbelauer et $a l^{7}$ also used AS-OCT in 138 eyes and found mean ACA of $28^{\circ}$ with a standard deviation of $16^{0}$. Again, this is not too dissimilar to the findings of this study despite all the differences that apply to the two studies in terms of study aims, methodology, et cetera. Naturally, the quantitative and graphical methodology applied in this paper (such as polar plots) to meridional measures of ACA via Scheimpflug photography could very easily be used for similar measures obtained through the use of different technologies such as AS-OCT or UBM.

The study by Rabsiler ${ }^{12}$ also used SP in 79 eyes with an older age group ( $46.6 \pm 16.8$ years $)$ and found mean ACA of $34.81 \pm 5.05^{\circ}$. This result is not too dissimilar to those of this study (see Table 2) especially for the superior quadrants of the three subjects involved. But, again, they ${ }^{12}$ were concerned with understanding ACA in multiple eyes and in a larger sample whereas this study involves short-term variation of ACA in a small number of eyes and thus the two studies were very different in terms of aims and design.

Another concern with Scheimpflug photography is that various image distortions occur with this digital and photographic method and instruments used may or may not have software or other approaches (such as tilting of the digital imaging sensor) that correct for some or all of these distortions. Such distortions could have an influence on issues such as repeatability and accuracy or validity of measurements. Besides AS-OCT or UBM, another alternative to Scheimpflug photography that has been suggested is high-resolution magnetic resonance imaging (HR-MRI) of the anterior segment of the eye ${ }^{24}$. HR-MRI has no distortions in comparison to Scheimpflug photography but produces lower resolution information or images 
and is not that easily accessible to optometrists or ophthalmologists and is, of course, a relatively costly procedure for patients.

Scheimpflug photography of the ACA is practical, reasonably repeatable, and easily and rapidly obtainable in a non-invasive manner. With further application of some of the methods, such as polar plots of meridional statistics, as used in this paper understanding short- or long-term variation of ACA is significantly simplified and enhanced. This should positively contribute towards more effective diagnosis and monitoring of treatment of ocular or other disorders that may influence the anterior segment of the eye. Possibly imaging or other technological improvements in SP might reduce or eliminate some of the suggested deficiencies relating to measurement of ACA. However, a great deal of further research remains necessary to extend our knowledge of the potential usefulness and advantages of these approaches to measurement of the ocular anterior chamber angle and to the many contributory influences to creating lesser or greater variation in the angular dimensions. Studies of menstrual-related or diurnal variation ${ }^{25}$ in the different angles of the anterior chamber would be two such topics for possible study. Variation in ACA with the ageing process or accommodation ${ }^{3}$ or relating to refractive state may be other productive areas for further investigation. Systemic or ocular disease that could impact on ACA could be studied and the nature of the healthy eye and its anterior chamber angles in different population groups could be more thoroughly understood through application of some of the approaches as used in this paper. Attempts should also be made to more clearly understand the inter-relationships and possible correlations that may exist between different variables such as ACA and axial anterior chamber depth ${ }^{26}$ or volume. Through this paper hopefully some new insights into the many complexities and uncertainties surrounding the ocular anterior chamber angle and its measurement via Scheimpflug photography may perhaps stimulate future study of this interesting and relatively unexplored topic.

\section{Acknowledgements}

L Geyser, E Schalkwyk and H Smit are thanked for their significant assistance towards subject selection and measurement of the necessary raw data for fur- ther analysis by the author. Thanks are also extended to the three subjects who participated in this study.

\section{References}

1. Lee DA, Brubaker RF, Ilstrup DM. Anterior chamber dimensions in patients with narrow angles and angle-closure glaucoma. Arch Ophthalmol 1984102 46-50.

2. Zarbin M, Chu D. Anterior chamber angle assessment techniques. Surv Ophthalmol 200853 250-273.

3. Drexler W, Baumgartner A, Findl ACA, Hitzenberger CK, Ferchere AF. Biometric investigation of changes in the anterior eye segment during accommodation. Vision Res 1997 37 2789-2800.

4. Congdon NG, Spaeth GL, Augsburger J, Klancnik J, Patel K, Hunter DG. A proposed simple method for measurement in the anterior chamber angle: Biometric gonioscopy. Ophthalmol 1999106 2161-2167.

5. Pavlin CJ, Harasiewicz K and Foster FS. Ultrasound biomicroscopy of anterior segment structures in normal and glaucomatous eyes. Am J Ophthalmol 1992113 381-389.

6. Xu L, Cao WF, Wang YX, Chen CX, Jonas JB. Anterior chamber depth and chamber angle and their associations with ocular and general parameters: The Beijing Eye Study. Am J Ophthalmol 2008145 929-936.

7. Wirbelauer C, Karandish A, Häberle H, Pham DT. Noncontact goniometry with optical coherence tomography. Arch Ophthalmol 2005123 179-185.

8. Kashiwagi K, Kashiwagi, Toda Y, Osada K, Tsumura T, Tsukahara S. A newly developed peripheral anterior chamber depth analysis system; principle, accuracy, and reproducibility. Br J Ophthalmol 200488 1030-1035.

9. Friedman DS, Gazzard G, Foster P, Devereux J, Broman A, Quigley H, Tielsch J, Seah S. Ultrasonographic biomicroscopy, Scheimpflug photography, and novel provocative tests in contralateral eyes of Chinese patients initially seen with acute angle closure. Arch Ophthalmol 2003121633 642.

10. Lackner B, Schmidinger G and Skorpik C. Validity and repeatability of anterior chamber depth measurements with Pentacam and Orbscan. Optom Vis Sci 2005 82 858-861.

11. Buehl W, Stojanac D, Sacu S, Drexler W, Findl ACA. Comparison of three methods of measuring corneal thickness and anterior chamber depth. Am J Ophthalmol 2006141 7-12.

12. Rabsilber TM, Khoramnia R and Auffarth GU. Anterior chamber measurements using Pentacam rotating Scheimpflug camera. J Cataract Refrac Surg 200632 456-459.

13. Shankar H, Taranath D, Santhirathelagan CT, Pesudovs K. Anterior segment biometry with the Pentacam: Comprehensive assessment of repeatability of automated measurements. J Cataract Refrac Surg 200834 103-113.

14. Emre S, Doganay S, Yologlu S. Evaluation of anterior segment parameters in keratoconic eyes measured with the Pentacam system. J Cataract Refrac Surg 200733 17081712. 
15. Nemeth G, Vajas A, Kolozsvari B, Berta A, Modis L. Anterior chamber depth measurements in phakic and psudophakic eyes: Pentacam versus ultrasound device. $J$ Cataract Refrac Surg 200632 1331-1335.

16. Kucumen RB, Yenerel NM, Gorgun E, Kulacoglu DN, Dinc UA, Alimgil ML. Anterior segment optical coherence tomography measurement of anterior chamber depth and angle changes after phacoemulsification and intraocular lens implantation. J Cataract Refrac Surg 200834 1694-1698.

17. Yamaguchi T, Negishi K, Yuki K, Saiki M, Nishimura R, Kawaguchi N, Tsubota K. Alterations in the anterior chamber angle after implantation of iris-fixated phakic intraocular lenses. J Cataract Refrac Surg 200834 1300-1305.

18. van Gool RD, Harris WF. Refractive variation under accommodative demand: curvital and scaled torsional variances and covariance across the meridians of the eye. Optom Vis Sci 199774 445-451.

19. van Gool RD. Refractive variation under accommodative demand. DPhil Thesis. Department of Optometry, Rand Afrikaans University, Johannesburg, South Africa, 2000.

20. Hill T, Lewicki P. Statistics methods and application: a comprehensive reference for science, industry and data mining. Tulsa: StatSoft, 2006 pp 41-56, 390.

21. Leung CK, Lam S, Tse RK, Chan W. Novel approach for anterior chamber angle analysis. Arch Ophthalmol 2006 124 1395-1401.

22. Baikoff G. Safety criteria for phakic intraocular lens implantation based on anterior optical coherence tomography. In: Anterior segment optical coherence tomography. Steinert RF, Huang D (Eds). New Jersey: SLACK Incorporated, 2008 pp123-124.

23. de Souza RF, Allemann N, Forseto A, Moraes Barros PS, Chamon W, Nosé W. Ultrasound biomicroscopy and Scheimpflug photography of angle-supported phakic intraocular lens. J Cataract Refrac Surg 200329 1159-1166.

24. Koretz JF, Strenk SA, Strenk LM, Semmlow JL. Scheimpflug and high-resolution magnetic resonance imaging of the anterior segment: a comparative study. J Opt Soc Am A 200421 346-354.

25. Read SA, Collins MJ, Iskander RD. Diurnal variation of axial length, intraocular pressure, and anterior eye biometrics. Invest Ophthalmol Vis Sci 200849 2911-2918.

26. Mathebula SD, Rubin A. Short-term variation of central corneal thickness and axial anterior chamber depth of healthy eyes using Scheimpflug photography via the Oculus Pentacam. S Afr Optom 200968 12-24. 